undoubtedly make the book longer, but would give the reader a much more comprehensive picture of the problem of drug misuse in the contemporary world.

The 14 chapters of the book give a description of the syndromes related to what the author considered to be the most prevalent drugs (depressants, alcohol, stimulants, opioids and other analgesics, cannabinols, hallucinogens and related drugs, glues, inhalants, and aerosols, over-the-counter drugs, prescription drugs, xanthines and nicotine), information on the drug combinations and the emergency problems. Finally, the book ends with a chapter on the rehabilitation of chronic drug users, which broadens the perspective of the earlier chapters focusing mainly on psychopharmacological aspects, with a brief, factual description of the psychosocial and other biological, naturopathy treatments.

At the end of each chapter is a detailed bibliography containing an average of more than a hundred of the most relevant items on the topic.

Schuckit's clinical guide provides practising doctors with an important conceptual framework and information for the solution of daily clinical problems.

J Gerevich

\section{Management mistakes in healthcare: identification, correction and prevention}

Edited by Paul B Hofman, Frankie Perry. Published by Cambridge University Press, Cambridge, 2005, \$95.00 (hardback), pp 255. ISBN 0-521-82900-3

Dick Davidson reminds us in the foreword to this important book that the public perception of the American hospital as a partner in its community, supporting "kindly Marcus Weldon MD" of 1970s television fame in the US, has changed. Although the nurses and doctors in the emergency room still seem heroic, the hospital seems more like a bureaucratic barrier getting in the way of "good people trying to help people". Systems are now in place in the US and the UK for the detection and prevention of clinical error, but the detection and prevention of healthcare managers' errors has not, until publication of this book, received the same attention.

The book aims to be a first step towards acknowledging and examining mistakes in healthcare management. Although their consequences are not as immediate as for clinical error, management errors may affect the health of whole communities. The book recognises that it is not easy to define management error. It offers some options here, emphasising a thorough review of evidence before a decision is made, and distinguishing error from intentional wrongdoing. The target audience is chief executives, senior managers and clinical managers (because clinical error, it argues, can become a management issue), and perhaps also risk managers, clinical governance staff (in the UK) and ethics staff (in the US). The book comprises six initial thought pieces followed by seven US-based case studies. The content is mainly relevant to US, but a reflective chapter on UK facilitates the transfer of some lessons from the case studies.

The thought pieces cover some sources and types of error (eg, of commission and omission), levels of disclosure, coping strategies for individual errors, methods of improving management performance, and systems and policies for managing management errors Other chapters look in detail at the dimensions of the context of managerial mistakes, and present alternative or complementary taxonomies of sources, types, and ways of disclosing, managing, correcting and preventing management mistakes. There is a reflection on the lessons from medical mistakes, and recognition that there is management responsibility for both the antecedents and the consequences of clinical error. A final reflective chapter considers accountability for management mistakes, suggesting that the "no-fault society may actually have some limits".

The case studies are generally lively and convincing. They cover the management response to medical error, management of a nursing shortage, problems with IT procurement, inept strategic planning in the development of a new hospital, mistakes in public relations after a death caused by equipmen failure, problems with clinical governance, specifically, in engaging the board in providing "loyal sceptic" ("iron sharpens iron") suppor for the CEO and an account of a failed hospita merger. Although the cases are all US based the UK review of selected cases highlights the UK national systems for reporting mistakes and sharing lessons, and points out that in the UK, chief executives of healthcare organisations are now legally accountable for mistakes made by their staff. The final chapter sums up the lessons learnt from both case studies and the thought chapters.

The book makes a commendable start in dealing with healthcare management error. It emphasises the need to be open and honest, alongside acknowledging that complete candour may not always be useful. It does not draw explicitly on concepts from organisational learning which could point to the links between robust systems for detecting and preventing error using single-feedback loop learning and double-feedback loop goal changing learning. An open learning culture-for example, using the method of Argyris and Senge, needs to include the possibility of innovation. The definitions of management mistakes are not fully convincing, because the nature of management evidence means that management mistakes can be made sense of in different ways from different perspectives, and this, the political dimension, is not much discussed. Some of the differences in principle between management and clinical mistakes are usefully dealt with. I would recommend this book for all senior healthcare managers interested in learning from mistakes.

A D Millard

\section{Bovine spongiform encephalopathy: risk, science and governance}

Authored by $\mathrm{P}$ van Zwanenberg, E Millstone Published by Oxford University Press, Oxford, 2005, £35.00 (hardback), pp 303. ISBN 0-19852581-8.

Over the past decade, there has been an increasing number of food alerts-bovine spongiform encephalopathy (BSE), dioxin Listeria, Salmonella-creating a genuine crisis of confidence among consumers in food safety and its governance. Of these food alerts, the
BSE one has been the worst, developing the characters of a real emergency.

van Zwanenberg and Millstone's book BSE: risk, science and governance provides a comprehensive analysis of the development of the BSE crisis, tracing its roots back to traditional UK agriculture and food policy-making. From these premises, the book analyses the course of events from the discovery of the first BSE case in November 1986 to the final failure that brought profound institutional changes in the UK, in the European Union and in several European countries. Particularly, the role of the relationships between science and public policy making in the development of the BSE crisis has been thoroughly analysed. The focus of the book is mainly the various lessons that can be learnt from the BSE saga. The history of BSE is presented as a paradigm of situations that require interaction between science and politics in a framework, where scientific uncertainties predominate.

The book first describes the role of science in public policy making and the different structures and approaches for science-based policy making after 1945 and up to the publication of the US NRC's Red Book of 1983, which states the paradigm adopted after the BSE crisis by the European Commission and the European Food Safety Authority for their policy-making process. In the subsequent chapter, the evolution of UK policy making on agriculture and foodrelated topics during the past two centuries is described. This chapter provides a basis for understanding the psychology of the Ministry of Agriculture, Forestry and Fisheries officials that led to the development of the BSE crisis. The next three chapters describe reactions to the detection of the first BSE cases, characterised by the imposition of a policy of secrecy at first, followed by seeking the help of advisory committees (Southwood Working Party, Tyrell Committee and Spongiform Encephalopathy Advisory Committee) to try to provide a justification for, and a masquerade to disguise, faulty policy judgements. The last chapters of the book, before the summary and conclusions, are about the BSE policy in continental Europe and the partial reform of food policymaking in the UK, the European Union and some European countries (France and Germany).

The analysis of the BSE policy in continental Europe is less complete and convincing than that carried out on the UK policy. The European reaction to the 1996 crisis, after the first cases of variant Creutzfeld-Jacob disease, is not analysed in all its aspects and consequences. In particular, the book does not deal with the testing of all bovine animals slaughtered for human consumption, adopted in the early 2000s. This was a clear over-reaction in contrast with the previous period that the authors properly define as "regulatory rigor mortis". This mass testing, presented to the public as a precautionary decision, an overkill from the scientific viewpoint, as it did not prevent a significant amount of infected animals from entering the human food chains, but was quite an effective way to overcome the attitude of European veterinary officials of overlooking the importance of BSE and to obtain widespread testing of animals in the categories at risk. This is another example of flawed policy decisions based on non-scientific motivations and concealed under the pretence of science-based policy making, which has characterised the entire BSE saga.

A Giovannini 